PHYSICAL REVIEW D 91, 122001 (2015)

\title{
Search for an emission line of a gravitational wave background
}

\author{
Atsushi Nishizawa* \\ Theoretical Astrophysics 350-17, California Institute of Technology, Pasadena, California 91125, USA \\ Naoki Seto \\ Department of Physics, Kyoto University, Kyoto 606-8502, Japan
}

(Received 8 March 2015; published 1 June 2015)

\begin{abstract}
In light of the history of research on an electromagnetic wave spectrum, a sharp emission line of gravitational wave background (GWB) would be an interesting observational target. Here we study an efficient method to detect a line GWB by correlating data of multiple ground-based detectors. We find that the width of frequency bin for coarse graining is a critical parameter, and, with the commonly used value $0.25 \mathrm{~Hz}$, the signal-to-noise ratio could be decreased by up to a factor of 6.6, compared with a finer width of $0.02 \mathrm{~Hz}$. By reanalyzing the existing data with a smaller bin width, we might detect a precious line signal from the early Universe.
\end{abstract}

DOI: 10.1103/PhysRevD.91.122001

PACS numbers: 04.80.Nn, 98.80.-k, 98.80.Cq

\section{INTRODUCTION}

Newton refurbished our understanding of the colors of visible light by sophisticatedly utilizing prisms, in addition to his extraordinary accomplishments on gravitation and classical mechanics. His sequential works on optics were summarized in Opticks published initially in 1704. But we had to wait for another $\sim 100$ years for significant experimental developments on electromagnetic wave spectra. In 1800, Herschel uncovered infrared components of a light ray. Around the same time, Wollaston (more systematically, Fraunhofer) discovered sharp absorption lines in the spectrum of sunlight. Moreover, the new element helium was identified in the solar corona by its emission lines detected at the total solar eclipse in 1868. However, the physical mechanism itself behind the observed sharp emission or absorption lines could be hardly clarified in the 1800s. Indeed, this conundrum was one of the primary driving forces toward the construction of quantum mechanics in the early 1900s.

On the other hand, the existence of a gravitational wave $(\mathrm{GW})$ is predicted by Einstein's general theory of relativity that was completed in 1915. As the gravitational interaction is very weak, GWs have not been directly observed yet. However, with the advent of powerful second-generation interferometric detectors, the first detection could be achieved shortly.

The high penetrating power of GWs could become quite advantageous to probe the early Universe. At present, our observational information before the big bang nucleosynthesis is severely limited [1]. Therefore, once primordial GWs are detected, they would provide us with invaluable clues to understand inflation and the subsequent reheating era [2-6] and high energy physics [7-10]. Also, GW

\footnotetext{
*anishi@caltech.edu
}

backgrounds (GWBs) bring us opportunities to test gravity theories [11-14]. To maximally extract the scientific outputs from the accumulated data of the interferometers, we should deliberate on methods of data analysis and thoroughly search for stochastic GWBs, not only for given theoretical predictions but also in model-independent manners.

In light of the aforementioned history of electromagnetic waves, it would be interesting to explore a sharp emission line of a cosmological GWB. Such a line GWB is not vital for the contemporary standard model of cosmology but is still allowed to exist. To create a line, there needs to be a sharply defined physical scale such as periodicity, boundaries, or discreteness. One example is large extra dimensions existing only in the early Universe and compactified later. GWs could resonate at a certain frequency if the topology of the extra dimensions is torus or is warped between branes, e.g. $[15,16]$. Another example is a massive GWB. If a graviton has tiny mass and the GWB is created by inflation, it is shown that, at the corresponding frequency to the graviton mass, a sharp peak on a GW spectrum appears at the present time [13].

Our purpose here is not predicting a GW spectrum in a specific cosmological scenario but investigating how these scenarios are constrained by GW observations in a modelindependent way. To this end, in this paper, we study how a line GWB looks in detector signals and suggest an efficient detection method by correlating data of multiple groundbased interferometers. We find that the width of frequency bin should be chosen carefully so that the size is much smaller than $0.1 \mathrm{~Hz}$. With the commonly used width $0.25 \mathrm{~Hz}$ [17], the signal-to-noise ratio could be reduced by up to a factor of 6.6 , compared with the width of $0.02 \mathrm{~Hz}$. Therefore, by reanalyzing the existing data, we might strike a precious line signal that has been buried in detector noises. 


\section{DOPPLER BROADENING OF GWS}

Throughout this paper, GWB is assumed to be isotropic in the cosmic microwave background (CMB) rest frame. As a simple but suggestive example, we first examine a deltafunction-like spectrum concentrating solely at a frequency $f_{\mathrm{r}}$ (cosmologically generated at the same comoving wavelength). Our study can be easily extended to a GWB with multiple lines. The effects of a line profile will be discussed later.

For a detector moving relative to the CMB frame with velocity $\vec{v}(t)$, the observed Doppler-shifted frequency $f(t, \hat{\Omega})=f_{\mathrm{r}}[1-\vec{v}(t) \cdot \hat{\Omega}]$ is anisotropic and depends on the propagation direction of a GW $\hat{\Omega}$. In this paper, we use the unit $c=1$ for the speed of light and also drop the terms of $\mathrm{O}\left(|\vec{v}(t)|^{2}\right)$ for the Doppler effect.

The relative velocity $\vec{v}_{\mathrm{s}}$ of the Solar System barycenter (SSB) to the CMB rest frame is toward the Galactic coordinate $(\ell, b)=\left(263.99^{\circ} \pm 0.14^{\circ}, 48.26^{\circ} \pm 0.03^{\circ}\right)$ with the magnitude $v_{\mathrm{s}} \equiv\left|\vec{v}_{\mathrm{s}}\right|=(1.230 \pm 0.003) \times 10^{-3}$ (corresponding to $369.1 \mathrm{~km} \mathrm{~s}^{-1}$ ) [18]. Below, for simplicity, we put $\vec{v}(t)=\vec{v}_{\mathrm{s}}$, neglecting corrections (at most $\lesssim 10 \%$ ) due to the velocity of the detector relative to the SSB. We also neglect minor relativistic effects such as the Sachs-Wolfe fluctuations. These would be reasonable approximations for our demonstration, and we can easily employ more sophisticated models in actual data analysis. Then we have

$$
f(t, \hat{\Omega})=f_{\mathrm{r}}\left[1-v_{\mathrm{s}} \cos \theta\right]
$$

with the angle $\theta$ between $\hat{\Omega}$ and $\vec{v}_{s}$. For a given observational frequency, the GW signals come from a ring on the sky as shown in Fig. 1. The total width of the Doppler broadening is given by

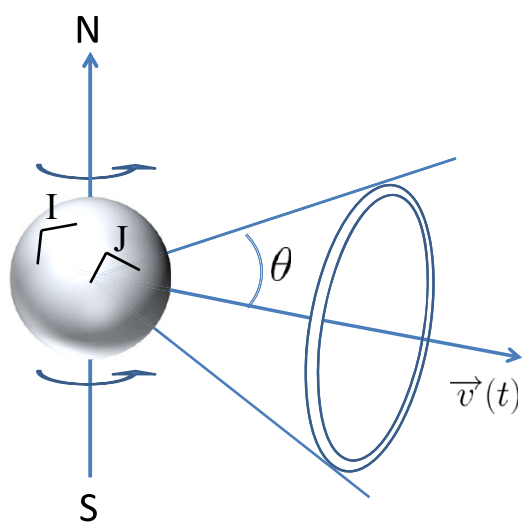

FIG. 1 (color online). Observation of a line GWB that is isotropic in the CMB rest frame. An observer (an interferometer on Earth) moves at the relative velocity $\vec{v}(t)$ and sees an isotropic background due to the Doppler effect. The observed frequency is identical along the ring across the sky with $\theta=\operatorname{const}[\theta$ is the angle between $\vec{v}(t)$ and the propagation direction of GW $\hat{\Omega}]$. The spin rotation of Earth generates diurnal modulation for the correlated signal of two interferometers $I$ and $J$.

$$
\delta f_{\mathrm{D}}=2 f_{\mathrm{r}} v_{\mathrm{s}} \sim 0.2\left(\frac{f_{\mathrm{r}}}{100 \mathrm{~Hz}}\right) \mathrm{Hz}
$$

The optimal band of ground-based interferometers is around $10-1000 \mathrm{~Hz}$. Meanwhile, by taking the Fourier transformation for an appropriate time segment (duration $T$ ) of data, we can realize a fine frequency resolution $\sim 10^{-3}(T / 1000 \mathrm{~s})^{-1} \mathrm{~Hz}$, unlike an electromagnetic-wave spectrum. But, as we discuss later, the spin rotation of Earth plays an important role, and this limits the duration $T$ for the coherent Fourier transformation.

\section{GW SIGNAL}

We briefly summarize the correlation analysis for an anisotropic GWB (not specific to a line GWB) [19] (see also $[20,21]$ for directional searches of GWBs). In the frequency space, the GW signal of a detector at the position $\vec{X}_{I}$ can be generally expanded as

$$
h\left(f, \vec{X}_{I}\right)=\sum_{A} \int_{S^{2}} d \hat{\Omega} \tilde{h}_{A}(f, \hat{\Omega}) e^{-2 \pi i f \hat{\Omega} \cdot \vec{X}_{I}} F_{I}^{A}(\hat{\Omega}) .
$$

Here, the label $A$ denotes the polarization mode $(A=+, \times)$ of a $\mathrm{GW}$, and $\tilde{h}_{A}(f, \hat{\Omega})$ is the Fourier mode of the GW in each polarization state. The factor $F_{I}^{A}$ is the antenna pattern function of the Ith $\mathrm{GW}$ detector and has a quadrupolar angular dependence. The power spectrum $S_{h}(f)$ for $\tilde{h}_{A}(f, \hat{\Omega})$ is given by the energy density of GWB $\Omega_{\mathrm{gw}}(f ; \hat{\Omega})$ (per logarithmic frequency interval per steradian) normalized by the critical density of the Universe as

$$
\Omega_{\mathrm{gw}}(f ; \hat{\Omega})=\frac{4 \pi^{2}}{3 H_{0}^{2}} f^{3} S_{h}(f ; \hat{\Omega}),
$$

where $H_{0}$ is the Hubble parameter and we use $H_{0}=70 \mathrm{~km} \mathrm{~s}^{-1} \mathrm{Mpc}^{-1}$.

The correlation analysis is an efficient method to distinguish a GWB signal from detector noises (see, e.g., [22-24]). With this method, we prepare data streams of two widely separated detectors (with independent noises) and take cross-correlations of their Fourier modes. For statistical amplification and coarse graining, we divide the Fourier space into multiple bins. Below, a bin is specified by the label $k$, and its central frequency and width are denoted by $f_{k}$ and $\delta f_{b}$, respectively. We intrinsically have $\delta f_{b}>T^{-1}$ due to the frequency resolution. Within each bin $k$, we take a summation of the correlated products. Its expectation value is written as [24]

$$
\mu_{k}=\frac{3 H_{0}^{2}}{8 \pi^{2}} \frac{T \delta f_{b}}{f_{k}^{3}} Z\left(f_{k}\right) .
$$

Here we defined the integral 


$$
Z\left(f_{k}\right) \equiv \int \frac{d \hat{\Omega}}{4 \pi} \Omega_{\mathrm{gw}}\left(f_{k} ; \hat{\Omega}\right) \gamma\left(f_{k}, \hat{\Omega}\right)
$$

and the direction-dependent overlap-reduction function

$$
\gamma(f, \hat{\Omega}) \equiv \sum_{A} F_{I}^{A}(\hat{\Omega}) F_{J}^{A}(\hat{\Omega}) \exp \left[2 \pi i f \hat{\Omega} \cdot \vec{d}_{I J}\right]
$$

with $\vec{d}_{I J}(t) \equiv \vec{X}_{I}(t)-\vec{X}_{J}(t)$ for two detectors $I$ and $J$. At the high frequency regime, the phase factor $\exp \left[2 \pi i f \hat{\Omega} \cdot \vec{d}_{I J}\right]$ depends strongly on the propagation direction $\hat{\Omega}$. In Eq. (5), we assumed to take a small bin width $\delta f_{b}$ so that the frequency dependence of relevant functions can be neglected within each bin. But this is not always the case, as we later discuss in detail.

Now we apply the above expressions specifically to the line GWB at a frequency $f_{\mathrm{r}}$ (defined in the CMB frame). From Eq. (1), we can write the observed spectrum as

$$
\Omega_{\mathrm{gw}}(f ; \theta)=\tilde{e}_{\mathrm{gw}} f_{\mathrm{r}} \delta\left[f-f_{\mathrm{r}}\left(1-v_{\mathrm{s}} \cos \theta\right)\right],
$$

where $\tilde{e}_{\mathrm{gw}}$ is the total energy of the line normalized by the critical density.

For a line GWB, we define the overlap-reduction function $\Gamma(f, u)$ that is obtained by integrating $\gamma(f, \hat{\Omega})$ across the ring directions at $u \equiv \cos \theta=$ const as

$$
\Gamma(f, u) \equiv \int_{0}^{2 \pi} \frac{d \phi}{2 \pi} \gamma(f, \hat{\Omega}) .
$$

This definition is different from the following standard one $\gamma_{\text {st }}(f)$ by an additional $u$ integral and the overall normalization:

$$
\gamma_{\mathrm{st}}(f) \equiv \frac{5}{8 \pi} \int_{-1}^{1} d u \int_{0}^{2 \pi} d \phi \gamma(f, \hat{\Omega}) .
$$

From Eqs. (6)-(9), we have

$$
Z\left(f_{k}\right)=\frac{\tilde{e}_{\mathrm{gw}} f_{\mathrm{r}}}{2} \int_{-1}^{1} d u \delta\left[f_{k}-f_{r}\left(1-v_{\mathrm{s}} u\right)\right] \Gamma\left(f_{k}, u\right) .
$$

Replacing the frequencies of slowly varying functions with $f_{\mathrm{r}}$ and putting $\Gamma\left(f_{k}\right) \equiv \Gamma\left(f_{\mathrm{r}}, u\left(f_{k}\right)\right)$, we obtain

$$
\mu_{k}=\frac{3 H_{0}^{2}}{16 \pi^{2}} \frac{T \tilde{e}_{\mathrm{gw}}}{f_{r}^{3} v_{\mathrm{s}}} \delta f_{b} \Gamma\left(f_{k}\right) .
$$

Note that the frequency $f$ and the sky angle $\theta$ (equivalently, $u$ ) are related by Eq. (1).

In Fig. 2, we show the directional overlap-reduction functions $\Gamma\left(f_{k}\right)$ observed by the two advanced-LIGO (aLIGO) interferometers for a line GWB at $f_{\mathrm{r}}=200 \mathrm{~Hz}$. The frequency dependence changes diurnally due to the spin rotation of Earth.

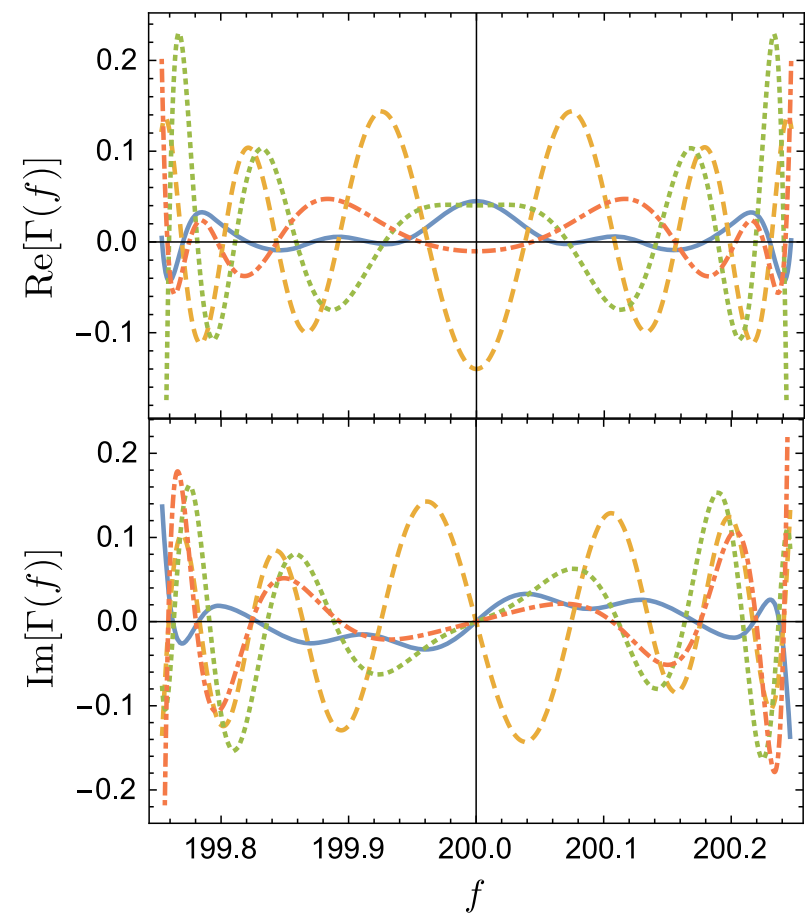

FIG. 2 (color online). Overlap-reduction function $\Gamma(f) \equiv$ $\Gamma\left(f_{\mathrm{r}}, u(f)\right)$ for two LIGO interferometers with $f_{\mathrm{r}}=200 \mathrm{~Hz}$. The observed frequency $f$ and the propagation direction $u \equiv$ $\cos \theta$ are related by Eq. (1). The upper panel is the real part, and the lower panel is the imaginary part. The overlap-reduction function is modulated by the spin rotation of Earth. Its shape is plotted at four different epochs: the fiducial time (blue, solid line), $3 \mathrm{~h}$ later (orange, dashed line), $6 \mathrm{~h}$ later (green, dotted line), and $9 \mathrm{~h}$ later (red, dot-dashed line). The characteristic frequency interval $\delta f_{c} \sim 0.1 \mathrm{~Hz}$ of the oscillations is given by Eq. (12).

The left and right edges of the curves are for GWs coming from the cold and hot spots of the CMB dipole and have large amplitudes. This is because the GW signals are hardly canceled by the $\phi$ integral in Eq. (9). In Fig. 2, we should also notice that the real (imaginary) part is symmetric (antisymmetric) around the intrinsic frequency $f_{\mathrm{r}}$. In relation to this structure, for an isotropic GWB (e.g., without a line component), the imaginary parts of the correlated signals vanish due to a cancellation. In contrast, for a line GWB, the overlap-reduction function $\Gamma(f)$ can have finite imaginary parts because of the anisotropy induced by the Doppler effect.

In Fig. 2, the wavy structure of the function $\Gamma(f)$ is mainly determined by the phase factor in Eq. (7). An oscillation occurs when the phase changes by $\delta \alpha \approx 2 \pi$, where $\alpha \equiv 2 \pi f_{\mathrm{r}} d_{I J} \cos \beta$ and $\cos \beta \equiv \hat{\Omega} \cdot \hat{d}_{I J}$. We also have $\delta(\cos \beta) \sim \delta(\cos \theta) \sim 1 /\left(f_{\mathrm{r}} d_{I J}\right)$. Then the characteristic frequency interval $\delta f_{c}$ for the oscillation is evaluated as

$$
\delta f_{c} \sim f_{\mathrm{r}} v_{\mathrm{s}} \delta(\cos \beta) \sim \frac{v_{\mathrm{s}}}{d_{I J}} \simeq 0.1\left(\frac{d_{I J}}{3000 \mathrm{~km}}\right)^{-1} \mathrm{~Hz} .
$$


Comparing this result with the Doppler width (2), the number of oscillations becomes larger for a higher frequency $f_{\mathrm{r}}$. For another detector pair more separated than aLIGOs $\left(d_{I J} \sim 3000 \mathrm{~km}\right)$, the characteristic interval $\delta f_{c}$ becomes smaller.

\section{SENSITIVITY TO A LINE GWB}

The correlated signal (11) would be suitable for a matched filtering analysis with the two fitting parameters $f_{\mathrm{r}}$ and $\tilde{e}_{\mathrm{gw}}$. To calculate the signal-to-noise ratio (SNR) of the line detection, we evaluate the variance of the correlated signal $\mu_{k}$. Here, for each Fourier mode, the noises of the two detectors are assumed to be independent and to have magnitudes much larger than those of the GW signals. Then, the variance of the correlation signal $\mu_{k}$ is mainly contributed by detector noises and is given by [24]

$$
\sigma_{k}^{2} \approx \frac{T}{8} \delta f_{b} P_{I}\left(f_{k}\right) P_{J}\left(f_{k}\right),
$$

with the one-sided noise spectra $P_{I}(f)$ and $P_{J}(f)$.

The correlation signal in Eq. (5) is a complex number, as mentioned before. From Eqs. (5), (11), and (13), the total squared SNR is evaluated as

$$
\rho^{2}=\sum_{k} \frac{\left|\mu_{k}\right|^{2}}{\sigma_{k}^{2}} \approx \frac{9 H_{0}^{4}}{32 \pi^{4}} \frac{T \tilde{e}_{\mathrm{gw}}^{2} \delta f_{b}}{f_{r}^{6} v_{s}^{2} P_{I}\left(f_{r}\right) P_{J}\left(f_{r}\right)} \sum_{k}\left|\Gamma\left(f_{k}\right)\right|^{2} .
$$

For a total observational duration $T_{\text {obs }}$ much longer than a day, we need to divide the data into time segments for taking Fourier transformation. But, in Eq. (14), we can effectively regard $T$ as the duration $T_{\text {obs }}$, after replacing $\left|\Gamma\left(f_{k}\right)\right|^{2}$ by its time average. Importantly, the total SNR $\rho$ does not depend on the details of the time segmentation, once we can take a sufficiently small bin $\delta f_{b}$ to resolve the function $\Gamma\left(f_{k}\right)$.

So far, we have assumed that the bin width $\delta f_{b}$ is much smaller than the characteristic frequency interval $\delta f_{c}$. If this is not the case, the coarse-grained signal $\left|\mu_{k}\right|$ decreases due to the averaging of the wavy structure around zero point, as understood from Fig. 2. Then, for Eq. (14), we should use the following integral:

$$
\mu_{k}=\frac{3 H_{0}^{2}}{16 \pi^{2}} \frac{T \tilde{e}_{\mathrm{gw}}}{f_{r}^{3} v_{\mathrm{s}}} \int_{\delta f_{b}} d f \Gamma(f)
$$

instead of the original one (11) that is valid only when the variation of the function $\Gamma(f)$ is negligible within each bin. Therefore, to realize a high SNR, we should set the bin width sufficiently smaller than the characteristic interval $\delta f_{c} \sim 0.1 \mathrm{~Hz}$. Nevertheless, the commonly used value is $\delta f_{b}=0.25 \mathrm{~Hz}$, e.g., in Ref. [17], and the sensitivity to a

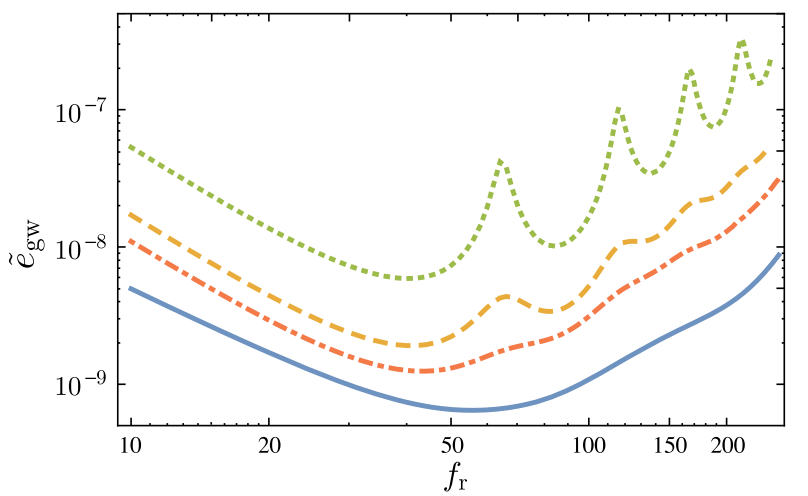

FIG. 3 (color online). Sensitivity to the normalized energy density $\tilde{e}_{\mathrm{gw}}$ of a line GWB. We assumed a 1 y observation with two aLIGO detectors and the detection threshold of $\rho=10$. The lower curve (blue, solid) is for the bin width $\delta_{b}=0 \mathrm{~Hz}$. The other curves from the top are with the bin width 2.5 (green, dotted), 0.25 (orange, dashed), and $0.1 \mathrm{~Hz}$ (orange, dotted-dashed), respectively.

line GWB might be decreased significantly. Below, we take a close look at this degradation.

In Fig. 3, the sensitivity to the normalized energy density $\tilde{e}_{\mathrm{gw}}$ is shown as a function of the line frequency $f_{\mathrm{r}}$. Here, we assumed a 1 y observation with two aLIGO detectors at the detection threshold of $\rho=10$. For their noise spectra $P_{I, J}(f)$, we use the fitting formulas given in Ref. [25]. In our study, the key parameter is the width of the frequency bin $\delta f_{b}$. For our demonstration here, we selected the following four widths: $0,0.1,0.25$, and $2.5 \mathrm{~Hz}$. The first one means a sufficiently small width (still satisfying $\delta f_{b}>T^{-1}$ ). To evaluate the SNR, we use Eq. (11) or (14) for this $0 \mathrm{~Hz}$ bin and Eq. (15) for the other three. Because, for a finite width, the sensitivity depends on the actual positions of the bins, we plotted the sensitivity averaged over their possible positions (by shifting boundaries of bins).

As shown in Fig. 3, the sensitivity decreases significantly for larger bin widths. This can be expected from the previous arguments on the cancellation of the wavy structure. The degradation of the sensitivity from $\delta_{b}=$ $0 \mathrm{~Hz}$ case, say, at $f_{\mathrm{r}}=70 \mathrm{~Hz}$, is by factors of 6.8 for $\delta f_{b}=0.25 \mathrm{~Hz}$ and 22 for $2.5 \mathrm{~Hz}$. Note that these ratios are independent of the detector noise spectra because the degradation is determined by the fine structure of the overlap-reduction functions. We also examined bin widths smaller than $\delta f_{c}=0.1 \mathrm{~Hz}$. For a line GWB with $10 \mathrm{~Hz}<f_{\mathrm{r}}<600 \mathrm{~Hz}$, the degradations (compared with $\delta f_{b}=0$ ) are within 1.1 for $\delta f_{b}=0.01 \mathrm{~Hz}, 1.2$ for $0.02 \mathrm{~Hz}$, 1.5 for $0.04 \mathrm{~Hz}, 3.7$ for $0.1 \mathrm{~Hz}$, and 7.9 for $0.25 \mathrm{~Hz}$. For example, to keep the loss of the sensitivity within $20 \%$, we should take a bin width $\delta f_{b}$ smaller than $0.02 \mathrm{~Hz}$, in contrast to the standard choice $0.25 \mathrm{~Hz}$.

In the opposite limit with $\delta f_{b} \gg \delta f_{\mathrm{D}}$, the $f$ integral in Eq. (15) is equivalent to posing an additional $u$ integral to 
Eq. (9). The result is proportional to the standard overlapreduction function $\gamma_{\mathrm{st}}(f)$ defined in Eq. (10). Indeed, in Fig. 3, the positions of the peaks (especially for the $2.5 \mathrm{~Hz}$ width) are around the zero points of the standard one $\gamma_{\mathrm{st}}(f)$ [24]. This is the reason we added the distinct results for the somewhat unrealistic choice of $\delta f_{b}=2.5 \mathrm{~Hz}$.

\section{DISCUSSIONS}

In the analysis above, we assumed a delta-function-like profile for a line GWB. Here we briefly comment on the effects of the intrinsic line width $\Delta f_{\mathrm{gw}}$ (now only considering $\delta f_{b} \ll \delta f_{c}$ ). This width $\Delta f_{\mathrm{gw}}$ should be compared with the Doppler broadening $\delta f_{\mathrm{D}}$ and the characteristic frequency interval $\delta f_{c}$. For a sharp line with $\Delta f_{\mathrm{gw}} \ll \delta f_{c}$, we can apply our previous arguments without change.

In the limit of a broad spectrum with $\Delta f_{\mathrm{gw}} \gg \delta f_{\mathrm{D}}$, a GWB at a certain observed frequency is contributed from the whole sky directions by the Doppler effect. There is no correspondence between an observed frequency $f$ and the angle $\theta$. In this case, by replacing $\Omega_{\mathrm{gw}}\left(f_{k} ; \hat{\Omega}\right)$ with $\Omega_{\mathrm{gw}}\left(f_{k}\right)$ and using Eqs. (6), (10), and (14), we have

$$
\rho^{2}=\frac{9 H_{0}^{4}}{50 \pi^{4}} T \delta f_{b} \sum_{k} \frac{\Omega_{\mathrm{gw}}^{2}\left(f_{k}\right) \gamma_{\mathrm{st}}^{2}\left(f_{k}\right)}{f_{k}^{6} P_{I}\left(f_{k}\right) P_{J}\left(f_{k}\right)} .
$$

This is the standard SNR formula for a broadband isotropic GWB [24]. Note that, for a fixed total energy density $\int d \ln f \Omega_{\mathrm{gw}}(f)=$ const (e.g., for the bound from the big bang nucleosynthesis [1] or CMB [26]), we can readily derive an approximate scaling relation $\rho \propto\left(\Delta f_{\mathrm{gw}}\right)^{-1 / 2}$ for the SNR.

In the intermediate case with $\delta f_{c}<\Delta f_{\mathrm{gw}}<\delta f_{\mathrm{D}}$, a GW signal at a certain observed frequency is contributed by GWs from a band region in the sky. Thus the GW signals are partially canceled, and the SNR is smaller than that for $\Delta f_{\mathrm{gw}}=0$ (for a fixed energy density).

When searching for a line GWB with real detectors, one might worry about confusion with various noise lines produced by instruments. However, we can clearly distinguish them by using their time dependences. In contrast to noise lines, a GW line is modulated due to the rotation and revolution of Earth, showing the characteristic profiles as illustrated in Fig. 2. Therefore, by appropriate matched filtering analyses, one could easily distinguish the true $\mathrm{GW}$ signals from artificial noises.

Although we have considered only an aLIGO pair, our study can be also applied to other planned detectors, such as LISA (sensitive around $10^{-4}-10^{-1} \mathrm{~Hz}$ ) and Einstein Telescope (ET) $(1-1000 \mathrm{~Hz})$. With the original LISA of the three-arm configuration [27], we can construct two noiseindependent data streams [28]. But, due to the symmetry of the system, their correlation identically vanishes for an isotropic GWB (as usually assumed). In contrast, a line GWB appears as a strongly anisotropic signal and could be detected, in principle, by the correlation. The situations are similar for ET with the proposed three-arm configuration [29]. However, eLISA is currently planned to have just two arms and can generate only a single data stream [30]. In this case, our correlation method no longer works. For a space mission like LISA, its third arm is essential to explore a line GWB.

\section{CONCLUSIONS}

We have studied how a line GWB looks in detector signals and suggested the method for efficiently searching for a line GWB by correlating data of multiple groundbased interferometers. Since the overlap-reduction function has fine wavy structures, the correlation signal could be canceled unless the bin width $\delta f_{b}$ is much smaller than the characteristic frequency interval $\delta f_{c}(\sim 0.1 \mathrm{~Hz}$ for the aLIGO pair). Nevertheless, for correlation analysis of GWBs, the commonly used width is $0.25 \mathrm{~Hz}$. By reanalyzing the existing data with a smaller width, we might actually uncover an important signal from the early Universe.

\section{ACKNOWLEDGMENTS}

A. N. is supported by JSPS Postdoctoral Fellowships for Research Abroad. N. S. is supported by JSPS (24540269) and MEXT (24103006).
[1] M. Maggiore, Phys. Rep. 331, 283 (2000).

[2] M. S. Turner, Phys. Rev. D 55, R435 (1997).

[3] L. A. Boyle and A. Buonanno, Phys. Rev. D 78, 043531 (2008).

[4] Y. Watanabe and E. Komatsu, Phys. Rev. D 73, 123515 (2006).

[5] R. Easther and E. A. Lim, J. Cosmol. Astropart. Phys. 04 (2006) 010.
[6] K. Nakayama, S. Saito, Y. Suwa, and J. Yokoyama, J. Cosmol. Astropart. Phys. 06 (2008) 020.

[7] M. Kamionkowski, A. Kosowsky, and M. S. Turner, Phys. Rev. D 49, 2837 (1994).

[8] C. Grojean and G. Servant, Phys. Rev. D 75, 043507 (2007).

[9] T. Damour and A. Vilenkin, Phys. Rev. D 71, 063510 (2005). 
[10] E. Fenu, D. G. Figueroa, R. Durrer, and J. Garcia-Bellido, J. Cosmol. Astropart. Phys. 10 (2009) 005.

[11] N. Seto, Phys. Rev. Lett. 97, 151101 (2006).

[12] A. Nishizawa, A. Taruya, K. Hayama, S. Kawamura, and M.-a. Sakagami, Phys. Rev. D 79, 082002 (2009).

[13] A. E. Gumrukcuoglu, S. Kuroyanagi, C. Lin, S. Mukohyama, and N. Tanahashi, Classical Quantum Gravity 29, 235026 (2012).

[14] A. Nishizawa and H. Motohashi, Phys. Rev. D 89, 063541 (2014).

[15] A. Chodos and S. Detweiler, Phys. Rev. D 21, 2167 (1980).

[16] S. S. Seahra, C. Clarkson, and R. Maartens, Phys. Rev. Lett. 94, 121302 (2005).

[17] J. Aasi et al. (LIGO Scientific Collaboration, VIRGO Collaboration), Phys. Rev. Lett. 113, 231101 (2014).

[18] N. Aghanim et al. (Planck Collaboration), Astron. Astrophys. 571, A27 (2014).

[19] B. Allen and A. C. Ottewill, Phys. Rev. D 56, 545 (1997).
[20] B. Abbott et al. (LIGO Scientific Collaboration), Phys. Rev. D 76, 082003 (2007).

[21] J. Abadie et al. (LIGO Scientific Collaboration), Phys. Rev. Lett. 107, 271102 (2011).

[22] N. Christensen, Phys. Rev. D 46, 5250 (1992).

[23] E. E. Flanagan, Phys. Rev. D 48, 2389 (1993).

[24] B. Allen and J. D. Romano, Phys. Rev. D 59, 102001 (1999).

[25] B. Sathyaprakash and B. Schutz, Living Rev. Relativity 12, 2 (2009)

[26] T. L. Smith, E. Pierpaoli, and M. Kamionkowski, Phys. Rev. Lett. 97, 021301 (2006).

[27] LISA Study Team, LISA Pre-Phase A Report, 1998, http:// lisa.nasa.gov/Documentation/FTR.pdf.

[28] T. A. Prince, M. Tinto, S. L. Larson, and J. Armstrong, Phys. Rev. D 66, 122002 (2002).

[29] M. Punturo et al., Classical Quantum Gravity 27, 084007 (2010).

[30] P. A. Seoane et al. (eLISA Collaboration), arXiv:1305.5720. 